\title{
Subdiagnóstico da Hipertensão Arterial em adultos quilombolas de região baiana,
}

\author{
Brasil \\ Underdiagnosis of Arterial Hypertension in quilombola adults from Bahia region, Brazil \\ Subdiagnóstico de Hipertensión Arterial en adultos de quilombola de la región de Bahía, Brasil
}

Recebido: 09/12/2020 | Revisado: 07/02/2021 | Aceito: 11/02/2021 | Publicado: 19/02/2021

Felipe Barreto de Matos

ORCID: https://orcid.org/ 0000-0002-0370-5597

Universidade Estadual do Sudoeste da Bahia, Brasil

E-mail: felipebm763@gmail.com

Lélia Renata Carneiro Vasconcelos

ORCID: https://orcid.org/0000-0003-1209-4799

Universidade Estadual do Sudoeste da Bahia, Brasil

E-mail: renatavcarneiro@gmail.com

Saulo Vasconcelos Rocha

ORCID: https://orcid.org/0000-0001-8655-5151

Universidade Estadual do Sudoeste da Bahia, Brasil E-mail: vasconcelosrocha82@ gmail.com

Ricardo Franklin de Freitas Mussi

ORCID: https://orcid.org/0000-0003-1515-9121 Universidade do Estado da Bahia, Brasil E-mail: rimussi@yahoo.com.br

\begin{abstract}
Resumo
A hipertensão arterial sistêmica (HAS) é uma doença crônica atrelada a fatores hereditários, econômicos e sociais, com alta prevalência na população negra brasileira. A negligência nas políticas públicas de saúde direcionadas a população quilombola gera importante exposição ao acometimento de HAS e seu subdiagnóstico. Neste sentido, a presente investigação objetiva analisar a prevalência e fatores associados ao subdiagnóstico da hipertensão arterial em adultos quilombolas de uma região baiana, Brasil. O estudo caracteriza-se como transversal, de base populacional, com amostra de 850 quilombolas da região baiana. Os dados foram coletados por meio de um questionário aplicado em forma de entrevista individual e avaliação da pressão arterial. Também foram incluídas as variáveis sociodemográficas, hábitos de vida e presença de hipertensão arterial. Na análise dos dados foram utilizados procedimentos da estatística descritiva e regressão de Poisson para determinação das razões de prevalência (RP) e intervalo de confiança de 95\% (IC95\%). A prevalência geral de HAS subdiagnosticada (HASsub) foi de 24,8\% e 29,2\% autorreferiram presença de HAS diagnosticada. Foi encontrada associação estatisticamente significante entre HASsub com sexo, escolaridade, tempo da pressão arterial (PA) mensurada, tabagismo e etilismo (p<0.05). Então, a HASsub está presente em 1 em cada 4 adultos quilombolas e está associada a fatores sociodemográficos (mulheres e idades avançadas), comportamento preventivo (PA mensurada em <1 ano) e relacionadas à situação de saúde (não ter excesso de peso). Esses achados demonstram a necessidade de práticas de atenção e promoção da saúde, além de mecanismos adotados para prevenção da doença.

Palavras-chave: Hipertensão; Fatores de risco; Comunidades vulneráveis; Grupo com ancestrais do continente africano; Inquérito epidemiológico.
\end{abstract}

\begin{abstract}
Systemic arterial hypertension (SAH) is a chronic disease linked to hereditary, economic and social factors, with a high prevalence in the black Brazilian population. Neglect in public health policies directed at the quilombola population generates important exposure to the onset of SAH and its underdiagnosis. In this sense, the present investigation aims to analyze the prevalence and factors associated with the underdiagnosis of arterial hypertension in quilombola adults from a Bahian region, Brazil. The study is characterized as cross-sectional, population-based, with a sample of 850 quilombolas from the Bahia region. Data were collected through a questionnaire applied in the form of an individual interview and blood pressure assessment. Sociodemographic variables, lifestyle and presence of arterial hypertension were also included. In the data analysis, descriptive statistics and Poisson regression procedures were used to determine the prevalence ratios (PR) and $95 \%$ confidence intervals $(95 \% \mathrm{CI})$. The general prevalence of underdiagnosed SAH (SAH sub) was $24.8 \%$ and $29.2 \%$ self-reported the presence of diagnosed SAH. A statistically significant association was found between HASsub with sex, education, measured blood pressure (BP) time, smoking and drinking ( $p<0.05$ ). So, HASsub is present in 1 in 4 quilombola adults and is associated with sociodemographic factors (women and advanced ages), preventive behavior (BP measured at $<1$ year) and related to the health situation (not being overweight).
\end{abstract}


These findings demonstrate the need for health care and health promotion practices, in addition to mechanisms adopted to prevent the disease.

Keywords: Hypertension; Risk factors; Vulnerable communities; Group with ancestors from the African continent; Epidemiological survey.

\begin{abstract}
Resumen
La hipertensión arterial sistémica (HSA) es una enfermedad crónica vinculada a factores hereditarios, económicos y sociales, con una alta prevalencia en la población brasileña negra. La negligencia en las políticas de salud pública dirigidas a la población de quilombola genera una exposición importante al inicio de la HSA y su diagnóstico insuficiente. En este sentido, la presente investigación tiene como objetivo analizar la prevalencia y los factores asociados con el diagnóstico insuficiente de hipertensión arterial en adultos de quilombola de una región de Bahía, Brasil. El estudio se caracteriza por ser transversal, basado en la población, con una muestra de 850 quilombolas de la región de Bahía. Los datos se recopilaron mediante un cuestionario aplicado en forma de entrevista individual y evaluación de la presión arterial. También se incluyeron variables sociodemográficas, estilo de vida y presencia de hipertensión arterial. En el análisis de datos, se utilizaron estadísticas descriptivas y procedimientos de regresión de Poisson para determinar las razones de prevalencia (RP) y los intervalos de confianza del 95\% (IC del 95\%). La prevalencia general de HSA subdiagnosticada (SAH sub) fue del 24,8\% y el 29,2\% autoinformaron la presencia de HSA diagnosticada. Se encontró una asociación estadísticamente significativa entre HASsub con sexo, educación, tiempo medido de presión arterial (PA), tabaquismo y consumo de alcohol ( $\mathrm{p}<0.05)$. Por lo tanto, HASsub está presente en 1 de cada 4 adultos de quilombola y se asocia con factores sociodemográficos (mujeres y edades avanzadas), conducta preventiva (presión arterial medida <1 año) y relacionada con la situación de salud (no tener sobrepeso). Estos resultados demuestran la necesidad de atención médica y prácticas de promoción de la salud, además de los mecanismos adoptados para prevenir la enfermedad.
\end{abstract}

Palabras clave: Hipertensión; Factores de riesgo; Comunidades vulnerables; Grupo con antepasados del continente africano; Encuesta epidemiológica.

\title{
1. Introdução
}

A hipertensão arterial sistêmica (HAS) é uma doença crônica não transmissível (DCNT) que acomete cerca de 1 bilhão de indivíduos em diferentes populações e localidades (NCD Risk Factor Collaboration, 2017), responsável por aproximadamente $70 \%$ das mortes no mundo, com impacto relevante nos países de média e baixa renda (Malta et al., 2017a). Além da HAS ser um fator de risco modificável, altamente relevante nas complicações desenvolvidas em enfermidades cardíacas e cerebelares (Go et al., 2013).

A exposição à comportamentos de risco como prática insuficiente de atividades físicas, alimentação inadequada, hábitos tabagista e consumo de bebida alcoólica além de fatores genéticos e a hereditariedade incrementam o risco de desenvolvimento da HAS (Brasil, 2016).

Destarte, ações de prevenção e tratamento têm se tornado alvos de políticas de saúde, especialmente em países subdesenvolvidos como o Brasil (Andrade et al., 2015), que conta com 35,7\% da população adulta hipertensa (Chor et al., 2015). Dentre os elementos que interferem na administração e tratamento da HAS, como determinantes comportamentais, estado sociodemográfico, nutrição e adesão à terapêutica farmacológica, deve ser considerada na formação e adoção de novas estratégias, com atuação de profissionais de saúde na avaliação periódica das pessoas (Morais et al., 2015; Barreto; Matsuda; Marcon, 2016).

Já foram evidenciadas associações entre raça-cor e desigualdades para o adoecimento, sendo as situações de fragilidade social e de saúde associada a questão étnico-racial (Bezerra et al., 2013; Pereira; Mussi, 2020). Neste sentido, populações negras apresentam prevalência de aproximadamente $41 \%$ de HAS (Chor et al., 2015) além de apresentarem maior probabilidade para seu acometimento (Bezerra et al., 2013; Andrade et al., 2015; Malachias et al., 2016).

A população negra necessita de um tratamento mais específico e intensivo da hipertensão, além de ter sido constatado maior propensão para modificação da pressão arterial (PA) em situações de consumo excessivo de sal em relação a população branca (Nadruz et al., 2017).

Baseado no levantamento da HAS segundo cor/raça pode-se suspeitar do maior subdiagnóstico entre pessoas 
pardas/pretas, remetendo ao abandono assistencial, concebendo extensas dificuldades, gerando mais óbitos; maior risco dessa população (geneticamente este problema pode se apresentar de forma mais acentuada); além da marginalização social, que gera a desassistência independente do diagnóstico final realizado (Brasil, 2016).

Dentre os perfis populacionais brasileiros, os quilombolas apresentam-se como grupo com importante quantitativo. Estas comunidades são definidas pela autoatribuição, a partir da dotação de relações territoriais específicas, com trajetória própria, e presunção na ancestralidade negra conectada com a resistência à opressão histórica sofrida (Mussi; Petroski, 2019).

Estudo de base populacional mostrou que aproximadamente $45,0 \%$ dos remanescentes de quilombos apresentaram-se hipertensos (Bezerra et al., 2013) contra 35,7\% identificada na população adulta brasileira (Chor et al., 2015). Neste sentido, visualizam-se os impactos da transição epidemiológica, acompanhada de maior demanda na atenção em saúde para o cuidado com o adoecimento crônico não-transmissível (Abreu et al, 2020), fortemente presente na população brasileira, com reflexos especialmente negativos na população negra quilombola.

A região Nordeste e o Estado da Bahia, com 61\% e 24\% respectivamente, apresentam o maior número de comunidades quilombolas certificadas do Brasil (Palmares, 2019). No entanto, essas regiões enfrentam dificuldades no acesso à saúde, condição socioeconômica restritiva e alto risco nutricional, portanto, com maior risco à saúde populacional e mais suscetíveis a subnotificação de agravos como a HAS (Bezerra et al., 2013). É relevante ressaltar que a subnotificação, resultante das limitações de infraestrutura, equipamentos e recursos humanos especializados, determina precariedade no monitoramento, análise e interpretação da situação epidemiológica populacional, dificultando a elaboração e execução de políticas e ações de saúde (Ossege et al, 2020).

Tendo em vista que raça-cor da pele interfere na HAS, a indicação de importante contingente de quilombolas acometidos, a carência na prestação de serviços em saúde e, o escasso conteúdo quanto à condição de saúde dessa população, inclusive quanto aos fatores associados e/ou predisponentes, que acabam restringindo a implementação de medidas de controle e prevenção do adoecimento, o objetivo deste estudo foi analisar prevalência e fatores associados ao subdiagnóstico da hipertensão arterial sistêmica em adultos quilombolas de uma região baiana, Brasil.

\section{Metodologia}

Trata-se de estudo de natureza quantitativa (Mussi et al, 2019b), do tipo transversal de base populacional que utiliza parte dos dados da pesquisa intitulada "Perfil Epidemiológico dos Quilombolas baianos",

O campo empírico de investigação foi a microrregião geográfica de Guanambi/Bahia, composta por 18 municípios, que contava com 42 quilombos contemporâneos rurais certificados até o ano de 2016 (Palmares, 2016), distribuídos em 10 destes municípios.

Diante da indisponibilidade de informações oficiais prévias relativas a quantidade de moradores dos quilombos desta microrregião baiana, a população foi estimada considerando 80 famílias por quilombo (Brasil, 2010), com dois adultos (pessoas com idade $\geq 18$ anos, de ambos os sexos) por família, indicando uma população de 6.720 adultos residentes nas comunidades quilombolas da região de Guanambi/Bahia.

O cálculo amostral adotou correção para população finita, prevalência de 50\% para desfecho desconhecido, intervalo de confiança de $95 \%$, erro amostral de cinco pontos percentuais, efeito de 1,5 vez para conglomerado em um estágio, acréscimo de $30 \%$ para recusas e $20 \%$ para perdas e confundimento, determinando amostra mínima de 818 sujeitos.

O desenho amostral consistiu em duas etapas: sorteio dos quilombos (conglomerado) e, logo depois, coleta censitária. Inicialmente foi realizado sorteio casual dos quilombos. Por meio das respectivas associações de moradores, três unidades recusaram participação da pesquisa, 14 que foram sorteadas permitiram atuação para a realização da pesquisa. 
Foram considerados elegíveis para participação no estudo todos os adultos residentes nos quilombos participantes. Nesse sentido, as associações de moradores informaram a presença de 1.025 adultos residentes durante o período das coletas que foram realizadas entre fevereiro e novembro de 2016. Todos foram convidados, sendo esclarecidos sobre os aspectos do estudo, garantindo igual probabilidade de participação. Compareceram voluntariamente às atividades e aceitaram participar por meio da assinatura ou fornecimento da impressão digital no Termo de Consentimento Livre e Esclarecido individual 850 quilombolas, compondo a amostra final. Não compareceram às atividades 17,07\% dos convidados, caracterizando as recusas.

Aqueles com deficiência cognitiva ou de comunicação avaliada como inapta foram excluídos das entrevistas. Acamados, amputados, engessados, grávidas e nutrizes com menos de seis meses foram excluídos das medidas antropométricas. As perdas foram definidas pela não realização de alguma medida ou ausência de resposta de alguma questão da entrevista.

A coleta de dados foi realizada por meio da aplicação de instrumento estruturado a partir de proposta validada para adultos quilombolas (Bezerra et al., 2014), sendo realizadas em sistema de mutirão, após as associações de moradores estabeleceram os dias para visitação. Todas as atividades foram desenvolvidas por equipes compostas por discentes e/ou profissionais da área de saúde de acordo suas competências, após preparação para sua respectiva função.

As medidas antropométricas (massa, estatura) foram determinadas conforme protocolo da International Society for the Advancement of Kinanthropometry (ISAK) (Stewart et al., 2011), em duplicata para igualdade, com terceira medida em caso de diferença, utilizando a mediana para análise. As coletas ocorreram em ambiente fechado, em momento único, pelo mesmo avaliador certificado ISAK, com os avaliados usando o mínimo de roupas e descalços.

A massa corporal foi mensurada em balança digital (Omron, modelo hbf-514c, com capacidade de $150 \mathrm{~kg}$ e precisão de 100g); a estatura foi coletada em estadiômetro metálico portátil (Sanny, modelo caprice, com resolução de 0,1mm). O erro técnico de medida (ETM) antropométrica intra-avaliador (Perini et al., 2005) foi de 0,12\% para estatura, 0,20\% para massa corporal, que indicam nível adequado das mensurações antropométricas.

A avaliação do \%G pelo método Bioimpedância foi realizada durante o período da manhã, antes do desjejum e determinado por balança validada (Omron hbf-514c, com capacidade 150Kg e precisão de 0,1\%) (Bosy-Westphal et al., 2008). As avaliações ocorreram em duplicata para igualdade, com terceira medida em caso de diferença, utilizando a mediana para análise. Anteriormente as mensurações foram verificadas abstinência alcoólica, de cafeína e de atividade física intensa nas últimas 24 horas. Os participantes também foram orientados a retirarem objetos metálicos e permanecerem em repouso durante cinco minutos antes dos testes.

A pressão arterial foi aferida com esfigmomanômetro semiautomático validado (Omron HEM-742INT) (Christofaro et al., 2009), em triplicata, após 10 minutos de repouso, com o entrevistado sentado, pés plantados no chão, braço esquerdo na altura do coração e a palma da mão voltada para cima, além de ter sido certificado se o paciente não: estava com bexiga cheia; exercício físico há pelo menos 60 minutos; fumou nos 30 minutos anteriores; ingeriu bebidas alcoólicas, café ou alimento (Malachias et al., 2016b). A média aritmética entre as medidas definiu o valor da pressão arterial.

As variáveis foram selecionadas de acordo com a literatura que indica as categorias e/ou fatores associados à hipertensão, abrangendo desta maneira: questões relativas aos hábitos de vida; medidas antropométricas; saúde autorreferida; e características sociodemográficas (Andrade et al., 2015; Malachias et al., 2016a; Malta et al., 2017b).

A variável dependente da presente análise é a HAS subdiagnosticada (HASsub), uma variável indicadora, resultado da interação entre duas variáveis da Pesquisa Nacional de Saúde. A primeira é a negativa se "Algum médico já lhe deu o diagnóstico de hipertensão arterial (pressão alta)?” associada a existência da segunda variável, pressão arterial sistólica (PAS) $\geq 140 \mathrm{mmHg}$ e/ou pressão arterial diastólica (PAD) $\geq 90$ mmHg (Chobanian et al., 2003). 
As características sociodemográficas são: sexo (feminino, masculino), faixa etária (adultos $>18$ anos e idosos $\geq 60$ anos), escolaridade ( $\leq 5$ anos, $>5$ anos), renda familiar (>1 salário mínimo, $\leq 1$ salário mínimo - conforme o valor vigente no ano de 2016), situação conjugal (com e sem cônjuge), situação laboral (sem remuneração, com remuneração).

As variáveis relativas ao hábito de vida, conforme questões da Pesquisa Nacional de Saúde validadas para adultos quilombolas (Bezerra et al., 2014), são: hábito tabagista (fumante, ex-fumante e nunca fumou), etilismo (consome bebida alcoólica regularmente: sim ou não), atividade física do tempo livre (nos últimos três meses, o(a) Sr.(a) praticou algum tipo de exercício físico ou esporte - fora fisioterapia? sim, não).

As variáveis relativas à situação de saúde foram: saúde autorreferida ('muito boa' e 'boa' agrupadas como positiva; 'regular'; e 'ruim' e 'muito ruim' agrupadas como negativa), presença de deficiência autorreferida (física, visual e/ou auditiva sim, não), conforme questões validadas para adultos quilombolas (Bezerra et al., 2014).

Também foram determinados tempo da PA mensurada $(\geq 1$ anos, $<1$ ano); excesso de peso pelo Índice de Massa Corporal (IMC) ( $>25 \mathrm{~kg} / \mathrm{m}^{2}$ definindo "excesso de peso", $<25 \mathrm{~kg} / \mathrm{m}^{2}$ para "sem excesso de peso") e obesidade por bioimpedância ( $>25 \%$ para homens e $30 \%$ para mulheres indicando 'obesidade', valores $<25 \%$ para homens e $<30$ para mulheres para "sem obesidade") (US Department of Health and Human Services, 2008).

A população foi caracterizada conforme as frequências, desvios padrão, valores mínimos, máximos e média.

Para análise de associação dos preditores com a HASsub foram estimadas razões de prevalências (RP) a partir da regressão de Poisson. Inicialmente foram verificadas as RP brutas. As variáveis que apresentarem p-valor $<0,20$ foram incluídas na análise múltipla. As variáveis que apresentou p-valor $<0,05$ no modelo saturado foram consideradas associadas ao desfecho. As magnitudes das associações foram estimadas pelo intervalo de confiança de $95 \%$. Todas as análises foram realizadas no programa The Statistical Package for Social Sciences, versão 22.0.

Esta análise utilizou dados do banco de estudo transversal de base populacional intitulado "Perfil Epidemiológico dos Quilombolas baianos", previamente autorizado pelo Comitê de Ética em Pesquisa com Seres Humanos da Universidade do Estado da Bahia, sob o parecer n 1.386.019/2016, com coletas desenvolvidas entre fevereiro e novembro de 2016.

\section{Resultados}

A HASsub foi prevalente em 24,8\% (IC95\%: 21,8:27,7) dos adultos quilombolas, enquanto 29,2\% (IC95\%: 26,2:32,3) citaram a presença de HAS diagnosticada.

As análises brutas (Tabela 1) indicaram associação estatisticamente significante $(\mathrm{p}<0,05)$ entre HASsub com sexo, escolaridade, tempo da PA mensurada, tabagismo e etilismo. Neste sentido, ser mulher quilombola diminui em aproximadamente 49,7\% a probabilidade de HAS não diagnosticada. Em relação à escolaridade indica-se que adultos quilombolas com >5 anos de estudo diminui em $23,7 \%$ a probabilidade da HASsub, e indivíduos que aferiram a pressão arterial $<1$ ano tem 30,4\% menor probabilidade de ter o subdiagnóstico. No mesmo sentido, nunca ter fumado e não consumir bebida alcoólica reduzem a possibilidade de HASsub em 38,9\% e 36,4\%, quando comparados aqueles fumantes e que relataram hábito etílico. 
Tabela 1. Razão de Prevalência (RP) da HASsub e seus intervalos de confiança de 95\% (IC 95\%) segundo variáveis sociodemográficas, estilo de vida e situação de saúde. Bahia, Brasil, 2016 (n=850).

\begin{tabular}{lllll}
\hline Variáveis & $\%(n)$ & $\%(n)$ HASsub & RP (IC95\%) & p-valor $^{1}$
\end{tabular}

Sexo

$$
\begin{aligned}
& \text { Masculino } \\
& \text { Feminino }
\end{aligned}
$$

Situação Conjugal

Com companheiro

Sem cônjuge

Grupo etário

Idoso

Adulto

Renda familiar

< salário mínimo

> salário mínimo

Escolaridade

$$
\begin{aligned}
& \leq 5 \text { anos } \\
& >5 \text { anos }
\end{aligned}
$$

Sem remuneração

Saúde Autorreferida

$$
\begin{aligned}
& \text { Negativa } \\
& \text { Regular }
\end{aligned}
$$

$41,4 \%$ (318)

$63,7 \%$ (522)

$36,3 \%$ (298)

$78,9 \%(575)$

$21,1 \%(154)$
$55,6 \%(114)$

$44,4 \%(91)$

$75,9 \%(148)$

$24,1 \%(47)$

$23,4 \%(48)$

$76,6 \%(157)$

0,777

$(0,592: 1,022)$

$(0,795: 1,405)$

$$
(0,397: 0,638)
$$

1

1,057

0,704

$$
\text { (05:1,405) }
$$$$
(0,592: 1,022)
$$

$77,9 \%$ (134)

$22,1 \%(38)$

1,059

0,721

$(0,774: 1,448)$

$65,0 \%$ (119)

$35,0 \%(64)$

$$
0,763
$$

$(0,584: 0,997)$

$62,9 \%(124)$

1

$37,1 \%(73)$

1,031

0,811

$(0,802: 1,326)$

0,071

0,721


$(0,590: 1,460)$

Positiva

Tempo da PA mesurada

$>1$ ano

$<1$ ano

$13,9 \%(114)$

$86,1 \%(704)$

$9,0 \%(72)$

$21,9 \%(175)$

Ex-fumante

Nunca fumou

$69,1 \%(553)$

$29,6 \%(242)$

$70,4 \%(576)$

Não bebe

Atividade física de tempo livre

Não

$\operatorname{Sim}$

Deficiência

Sim

Não

Índice de Massa Corporal

Com excesso de peso

Sem excesso de peso
$62,8 \%(515)$

$37,2 \%(305)$

$53,0 \%(434)$

$47,0 \%(385)$

$60,8 \%(490)$

$39,2 \%(316)$

$39,2 \%(76)$

$62,9 \%(124)$

$37,1 \%(73)$

$57,9 \%(117)$

$42,1 \%(85)$
1,145

0,542

$(0,740: 1,773)$

$18,9 \%(37)$

1

0,696

0,017

$(0,517: 0,937)$

1

0,728

0,115

$(0,490: 1,080)$

0,611

0,005

$(0,433: 0,863)$

$39,8 \%(78)$

1

0,636

$<0,001$

$(0,498: 0,811)$

$60,8 \%$ (118)

1

0,999

0,992

$(0,777: 1,284)$

1

0,994

0,963

$(0,773: 1,279)$

$\%$ de gordura 


\begin{tabular}{|c|c|c|c|c|}
\hline Obeso & $63,0 \%(505)$ & $60,1 \%(119)$ & 1 & \\
\hline Sem obesidade & $37,0 \%(296)$ & $39,9 \%(79)$ & 1,133 & 0,320 \\
\hline
\end{tabular}

n (\%): quantidade de sujeitos; \% com HASsub: percentual HASsub; RP: Razão de prevalência; IC95\%: intervalo de confiança de $95 \%$. Fonte: Dados da pesquisa.

Após a análise multivariada (Tabela 2) permaneceram independentemente associadas à HASsub: sexo, tempo da última PA mensurada e IMC. Logo, ser quilombola do sexo feminino diminui em 55,5\% a probabilidade para a HASsub. No mesmo sentido, ter mensurado a $\mathrm{PA}$ em período $<1$ ano e não ter excesso de peso corporal diminuem em respectivamente $28,8 \%$ e $31,0 \%$, a probabilidade do desfecho negativo analisado.

Tabela 2. Análise de regressão de Poisson múltipla dos fatores associados à HASsub em adultos quilombolas. Bahia, Brasil, 2016.

\begin{tabular}{|c|c|c|c|c|}
\hline Variáveis & RP * (IC95\%) & p-valor & RP ** (IC95\%) & p-valor \\
\hline \multicolumn{5}{|l|}{ Sexo } \\
\hline Masculino & 1 & & 1 & \\
\hline Feminino & $0,505(0,379: 0,672)$ & $<0,001$ & $0,445(0,354: 0,584)$ & $<0,001$ \\
\hline \multicolumn{5}{|l|}{ Grupo etário } \\
\hline Idoso & 1 & & & \\
\hline Adulto & $0,823(0,592: 1,145)$ & 0,248 & & \\
\hline
\end{tabular}

Escolaridade

\begin{tabular}{|c|c|}
\hline$\leq 5$ anos & 1 \\
\hline 5 anos & $0,932(0,696: 1,249)$ \\
\hline
\end{tabular}

Tempo da PA mensurada

$$
\begin{aligned}
& \geq 1 \text { ano } \\
& <1 \text { ano }
\end{aligned}
$$$$
0,686(0,509: 0,923)
$$

0,013

$0,712(0,536: 0,946)$

Tabagismo

Fumante

Ex-fumante

Nunca fumou

Etilismo

Bebe

Não bebe 
Índice de Massa Corporal

Com excesso de peso

Sem excesso de peso
1

$0,686(0,524: 0,897)$

0,006

$0,690(0,538: 0,885)$

0,003

RP: Razão de prevalência; IC95\%: intervalo de confiança de 95\%; * Regressão de Poisson ajustada por todas as variáveis que entraram no modelo; ** Regressão de Poisson múltipla conforme as variáveis que permaneceram no modelo. Fonte: Dados da pesquisa.

\section{Discussão}

Os resultados do presente estudo mostraram que aproximadamente $1 / 4$ dos adultos quilombolas podem estar com HASsub. Indivíduos do sexo feminino, o fato de ter mensurado a PA em período $<1$ ano e não ter excesso de peso corporal diminuem a probabilidade do desfecho negativo analisado.

Inquérito desenvolvido em comunidades quilombolas de Vitória da Conquista/Bahia identificou que aproximadamente metade dos adultos apresentavam HASsub (Bezerra et al., 2015), índice maior que o encontrado na presente investigação. Uma explicação plausível para a recorrência de HASsub na população quilombola é a baixa disponibilidade, ou mesmo ausência, de serviços de saúde pública que desenvolvam ações de cuidado, diagnóstico e manejo do processo saúde doença na população negra (Bezerra et al., 2013; Pereira, Mussi, Rocha, 2020; Pereira; Mussi, 2020; Almeida et al., 2019).

No mesmo sentido, o acesso aos serviços de saúde é fundamental para prevenção do agravo de enfermidades. Por exemplo, estudo com quilombolas de município baiano identificou pré-hipertensão prevalente em 30\% da população (Bezerra et al., 2017), situação que sem os cuidados em saúde adequados evoluirão rapidamente para quadros clínicos hipertensivos mais graves.

Assim como identificado na presente análise, outros estudos também identificaram associação de situações hipertensivas com o sexo masculino, seja quanto à ocorrência de HAS (Silva; Petroski; Peres, 2012; Silva et al., 2016), préhipertensão (Silva; Petroski; Peres, 2012) e HASsub (Bezerra et al., 2015). Estima-se que essa associação pode estar relacionada ao diferencial de comportamentos de riscos relacionados à saúde entre homens e mulheres. As mulheres são habitualmente mais predispostas a irem ao médico, o que diminui substancialmente a possibilidade de serem subdiagnosticadas com alguma doença.

Utiliza-se da mesma justificativa para respaldar o tempo de aferição da PA $<1$ ano ter possibilidade 30,4\% menor de ser subdiagnosticado, enquanto homens apresentam maiores prevalências de pré-hipertensão arterial, por serem grupos mais expostos a fatores de risco do que os do sexo feminino, principalmente no que se refere ao estilo de vida (Silva; Petroski; Peres, 2012).

Outro aspecto importante identificado no presente estudo foi que a redução da escolaridade aumenta a probabilidade de exposição a HAS. Esta situação corrobora a compreensão da interação positiva entre educação, de maneira geral e, mais especificamente, das práticas educacionais específicas e a prevenção do adoecimento e promoção da saúde (Mussi et al., 2016; Mussi et al., 2019).

No entanto, estudos mostraram que comunidades quilombolas são frequentemente mais predispostas a vulnerabilidade social (Bezerra et al., 2013, 2014), inclusive quanto a baixa escolarização (Teixeira Mussi, Mussi, Araújo, 2020), e que mulheres com menos anos de escolaridade apresentaram um aumento considerável na prevalência de hipertensão arterial (Hartmann et al., 2007). Este cenário é preocupante porque após período de crescimento no acesso a escolarização por grupos historicamente negligenciados, o final da década de 2010 é marcada por uma retração nesta perspectiva (Ribeiro et al, 2018), o que pode agravar a situação de vulnerabilidade social e em saúde nesta população.

Quanto ao estilo de vida, foi identificado que o tabagismo se associou a HASsub na população investigada. As relações entre o tabagismo e a HAS provém de uma complexa interação entre fatores hemodinâmicos, sistema nervoso autonômico e 
múltiplos mediadores vasoativos causadores da disfunção endotelial (Sousa, 2015). Apesar de seus reconhecidos malefícios, o tabagismo figura como prática recorrente entre pessoas hipertensas (Melo et al, 2020) como verificado entre os adultos quilombolas, mesmo que em situação de HAS subdiagnosticada.

Em relação ao etilismo, não consumir bebida alcoólica reduz a possibilidade de HASsub em 36,4\% na população quilombola. Em contexto similar, investigação conduzida por Julião e Guimarães (2018) mostrou que indivíduos que reportaram consumo nocivo de álcool têm $20 \%$ mais chance de estarem subdiagnosticados com hipertensão, apresentando-se como grupo de risco.

A ação do álcool sobre a pressão arterial é divergente e inconclusiva na literatura, porém acredita-se que o álcool atua no sistema renina-angiotensinaaldosterona (SRAA) e a estimulação simpática ou adrenal, ambos acometendo os níveis de hormônios antidiurético, cortisol e o mecanismo barorreflexo (Souza, 2014), gerando a sua ingestão crônica e nociva a aumentos consistentes da PA (Malachias et al., 2016a).

Outra associação identificada no presente estudo foi entre o excesso de peso e a presença de HASsub. Em adultos quilombolas, já foi documentada a importância do excesso de peso para o aumento da PA (Mussi, Queiroz e Petroski, 2018) e a obesidade associada à prevalência da HAS (Santos et al, 2020).

Neste sentido, o acréscimo do peso corpóreo correlaciona-se com exacerbação e desdobramento da HAS, sua alta prevalência em obesos é atribuída a resistência à insulina, principalmente em sujeitos que possuem excesso de adiposidade no abdome que gera ativação no sistema nervoso autônomo e consequente aumento da resistência vascular periférica e da PA (Monteiro et al., 2004; Costa, Deus, Alves, 2020).

Metodologicamente a presente análise não permite afirmar se os fatores associados determinam ou são determinados pelo subdiagnóstico, característica inerente aos estudos transversais. Outra limitação emerge da ausência de elementos que demonstrem a existência e/ou influência das condições gênicas na prevalência do subdiagnóstico e seus respectivos componentes nestas comunidades.

Dentre as vantagens do estudo cita-se o rigor metodológico na utilização de equipamentos e instrumentos previamente validados para investigações obtenção da PA e das medidas antropométricas; a adoção de questionário antecipadamente validado para adultos quilombolas; a representatividade de adultos residentes em quilombos de toda uma região estadual, mesmo diante da complexidade logística; e, o potencial colaborativo para a ampliação e/ou correção da efetividade das políticas públicas relativas à prevenção dos fatores de risco à saúde, tão importante para população negra brasileira.

\section{Conclusão}

A HASsub está presente em 1 em cada 4 adultos quilombolas, associada a fatores sociodemográficos (mulheres e maiores idades), comportamento preventivo (PA mensurada em $<1$ ano) e relacionadas à situação de saúde (não ter excesso de peso), demonstra a necessidade de práticas de atenção e promoção da saúde, além de mecanismos adotados para prevenção da doença, desta maneira resultando com o declínio do quadro epidemiológico.

É relevante destacar a carência de dados epidemiológicos específicos referentes à comunidade quilombola nos sistemas de informações, reitera a importância de estudos com esse grupo populacional para assim implementar estratégias para promoção e prevenção da HAS, objetivando o diagnóstico precoce, tratamento contínuo, controle da pressão arterial e dos fatores de risco associados que certamente influenciaram positivamente nas condições de vida e saúde dessa comunidade.

Nesse sentido recomenda-se a realização de mais estudos de rastreamento dessa condição entre a população quilombola nas diferentes regiões do país, com a inclusão de investigações que utilizem desenhos longitudinais. Essas ações possibilitarão uma melhor compreensão da HAsub além de estabelecerem relações de causalidade que poderão contribuir para o maior conhecimento acerca dessa temática. 


\section{Agradecimentos}

Agradecemos às Associações de Moradores das Comunidades Quilombolas que autorizaram as visitações para o desenvolvimento do estudo e colaboraram com a logística local das coletas.

\section{Referências}

Abreu, L. C. C., Silva, C. S. C. e, Santos, K. H. M. R. dos, \& Conceição, S. dos S. (2020). Educação interprofissional em saú de e seu impacto na atenção integral. Cenas Educacionais, 3, e8869.

Andrade, S. S. de A., Stopa, S. R., Brito, A. S., Chueri, P. S., Szwarcwald, C. L., \& Malta, D. C. (2015). Prevalência de hipertensão arterial autorreferida na população brasileira: análise da Pesquisa Nacional de Saúde, 2013. Epidemiologia e Serviços de Saúde, 24(2), $297-304$.

Barreto, M. da S., Matsuda, L. M., \& Marcon, S. S. (2016). Factors associated with inadequate blood pressure control in patients of primary care. Escola Anna Nery, 20(1), 114-120.

Bezerra, V. M., Andrade, A. C. de S., César, C. C., \& Caiaffa, W. T. (2013). Comunidades quilombolas de Vitória da Conquista, Bahia, Brasil: hipertensão arterial e fatores associados. Cadernos de Saúde Pública, 29(9), 1889-1902.

Bezerra, V. M., Medeiros, D. S., Gomes, K. O., Souzas, R., Giatti, L., Steffens, A. P., Kochergin, C. N., Souza, C. L., Moura, C. S., Soares, D. A., Santos, L. R. C. S., Cardoso, L. G. V., Oliveira, M. V., Martins, P. C., Neves, O. S. C., \& Guimarães, M. D. C. (2014). Inquérito de Saúde em Comunidades Quilombolas de Vitória da Conquista, Bahia, Brasil (Projeto COMQUISTA): aspectos metodológicos e análise descritiva. Ciência \& Saúde Coletiva, 19 (6), $1835-1847$.

Bezerra, V. M., Andrade, A. C. S., César, C. C., \& Caiaffa, W. T. (2015). Unawareness of hypertension and its determinants among 'quilombolas' (inhabitants of 'quilombos' - hinterland settlements founded by people of African origin) living in Southwest Bahia, Brazil. Ciência \& Saúde Coletiva, 20(3), 797-807.

Bezerra, V. M., Andrade, A. C. S., Medeiros, D. S. de, \& \& Caiaffa, W. T. (2017). Pré-hipertensão arterial em comunidades quilombolas do sudoeste da Bahia, Brasil. Cadernos de Saúde Pública, 33(10), e00139516.

Almeida, C. B. de, Santos, A. S. dos, Vilela, A. B. A., \& Casotti, C. A. (2019). Reflexão sobre o controle do acesso de quilombolas à saúde pública brasileira. Avances en Enfermería, 37(1), 92-103.

Bosy-Westphal, A., Later, W., Hitze, B., Sato, T., Kossel, E., Gluer, C. C., Heller, M., Muller, M. J. (2008). Accuracy of Bioelectrical Impedance Consumer Devices for Measurement of Body Composition in Comparison to Whole Body Magnetic Resonance Imaging and Dual X-Ray Absorptiometry. Obesity Facts, $1(6), 319-324$.

Brasil. (2016). Ministério da Saúde. Secretaria de Gestão Estratégica e Participativa. Departamento de Articulação Interfederativa. Temático Saúde da População Negra. Brasília, DF.

Brasil. (2010). Secretaria de Políticas de Promoção da Igualdade Racial da Presidência da República (SEPPIR). Comunidades Quilombolas Brasileiras Regularização Fundiária e Políticas Públicas,

Chobanian, A. V., Bakris, G. L., Black, H. R., Cushman, W. C., Green, L. A., Izzo, J. L. Jr., Jones, D. W., Materson, B. J., Oparil, S., Wright, J. T. Jr., Roccella, E. J., National Heart, Lung, and Blood Institute Joint National Committee on Prevention, Detection, Evaluation, and Treatment of High Blood Pressure, National High Blood Pressure Education Program Coordinating Committee. (2003). The Seventh Report of the Joint National Committee on Prevention, Detection, Evaluation, and Treatment of High Blood Pressure: The JNC 7 Report. Jama, 289(19), 2560-2572.

Chor, D., Ribeiro, A. L. P., Carvalho, M. S., Duncan, B. B., Lotufo, P. A., Nobre, A. A., Aquino, E. M., Schmidt, M. I., Griep, R. H., Mdel, C. M., Barreto, S. M., Passos, V. M., Benseñor, I. J., Matos, S. M., Mill, J. G. (2015). Prevalence, Awareness, Treatment and Influence of Socioeconomic Variables on Control of High Blood Pressure: Results of the ELSA-Brasil Study. Plos One, 10(6), p. e0127382.

Christofaro, D. G. D., Fernandes, R. A., Gerage, A. M., Alves, M. J., Polito, M. D., \& Oliveira, A. R. de. (2009). Validação do monitor de medida de pressão arterial Omron HEM 742 em adolescentes. Arquivos Brasileiros de Cardiologia, 92(1), 10-15.

Costa, G. D. da, Deus, R. M. L. de, \& Alves, W. dos S. (2020). Estudo epidemiológico da prevalência simultânea de hipertensão e diabetes de pacientes cadastrados no Hiperdia em uma cidade do estado do Piauí. Research, Society and Development, 9(2), e192922163.

Go, A. S., Mozaffarian, D., Roger, V. L., Benjamin, E. J., Berry, J. D., Borden, W. B., Bravata, D. M., Dai, S., Ford, E. S., Fox, C.S., Franco, S., Fullerton, H. J., Gillespie, C., Hailpern, S. M., Heit, J. A., Howard, V. J., Huffman, M. D., Kissela, B. M., Kittner, S. J., Lackland, D. T., Lichtman, J. H., Lisabeth, L. D., Magid, D., Marcus, G. M., Marelli, A., Matchar, D. B., McGuire, D. K., Mohler, E. R., Moy, C. S., Mussolino, M. E., Nichol, G., Paynter, N. P., Schreiner, P. J., Sorlie, P. D., Stein, J., Turan, T. N., Virani, S. S., Wong, N. D., Woo, D., \& Turner, M. B. and on behalf of the American Heart Association Statistics Committee and Stroke Statistics Subcommittee. (2013). Heart Disease and Stroke Statistics-2013 Update: A Report From the American Heart Association. Circulation, 127(1), 143-152.

Hartmann, M., Dias-da-Costa, J. S., Olinto, M. T. A., Pattussi, M. P., \& Tramontini, Â. (2007). Prevalência de hipertensão arterial sistêmica e fatores associados: um estudo de base populacional em mulheres no Sul do Brasil. Cadernos de Saúde Pública, 23(8), 1857-1866.

Julião, N., Guimarães, R. (2018). Fatores associados ao subdiagnóstico de hipertensão na população brasileira: Um estudo com base na Pesquisa Nacional de Saúde (2013). In: Anais do XXI Encontro Nacional de Estudos Populacionais, Poços de Caldas, MG.

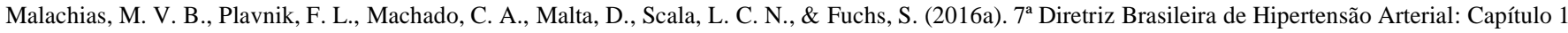
- Conceituação, Epidemiologia e Prevenção Primária. Arquivos Brasileiros de Cardiologia, 107(3, Suppl. 3), 1-6. 
Malachias, M. V. B., Gomes, M. A. M., Nobre, F., Alessi, A., Feitosa, A. D., \& Coelho, E. B. (2016b). $7^{\text {a }}$ Diretriz Brasileira de Hipertensão Arterial: Capítulo 2 - Diagnóstico e Classificação. Arquivos Brasileiros de Cardiologia, 107(3, Suppl. 3), 7-13.

Malta, D. C., Bernal, R. T. I., Lima, M. G., Araújo, S. S. C. de, Silva, M. M. A. da, Freitas, M. I. de F., \& Barros, M. B. de A. (2017a). Doenças crônicas não transmissíveis e a utilização de serviços de saúde: análise da Pesquisa Nacional de Saúde no Brasil. Revista de Saúde Pública, 51(Suppl. 1), 4s.

Malta, D. C., Bernal, R. T. I., Andrade, S. S. C. de A., Silva, M. M. A. da, \& Velasquez-Melendez, G. (2017b). Prevalência e fatores associados com hipertensão arterial autorreferida em adultos brasileiros. Revista de Saúde Pública, 51(Suppl. 1), 11s.

Melo, L. D. de, Jeremias, J. da S., Shubo, A. F. M. F., Taroco, F. E., Spindola, T., Gomes Filho, W., Ferreira, B. S. B., \& Barbosa, D. J. (2020). Tabagismo, Hipertensão Arterial Sistêmica e Pandemia da COVID-19: uma Análise Psicanalítica Freudiana. Research, Society and Development, 9 (11), e57891110240.

Monteiro, C. A., Conde, W. L., Lu, B., Popkin, B. M. (2004). Obesity and inequities in health in the developing world. International Journal of Obesity, 28(9), 1181-1186.

Morais, P. C. A., Moreira, R. P., Lima, P. A. de, Silva, M. das G. F., Ferreira, J. D. F., Rouberte, E. S. C. (2015). Blood pressure, heart diseases and lifestyles of elderly.

Revista da Rede de Enfermagem do Nordeste, 16(5), 722-730.

Mussi, R. F. F., José, H. P. M., Azevedo, D. P., Amorim, A. M., Petroski, E. L. (2019). O ensino da antropometria na escola: uma proposta na educação em saúde. Cenas Educacionais, 2(1):14-8.

Mussi, R. F. F., Freitas, D. A., Amorim, A. M., Petroski, E. L. (2016). Formação em educação física e a saúde na escola. In: (org.) Farias GO, Nascimento JV. Educação, saúde e esporte: novos desafios à Educação Física. Ilhéus: Editus.

Mussi, R. F. F., Queiroz, B. M., Petroski, E. L. (2018). Excesso de peso e fatores associados em quilomboras do médio São Francisco baiano, Brasil. Cienc saude coletiva, 23(4):1193-1200.

Mussi, R. F. F., Teixeira Mussi, L. M. P., Assunção, E. T. A. \& Nunes, C. P. (2019b). Pesquisa Quantitativa e/ou Qualitativa: distanciamentos, aproximações e possibilidades. Revista Sustinere, 7(2), 414-430.

Nadruz Jr., W., Claggett, B., Henglin, M., Shah, A. M., Skali, H., Rosamond, W. D., Folsom, A. R., Solomon, S. D., Cheng, S. (2017). Racial Disparities in Risks of Stroke. New England Journal Of Medicine, 376(21), 2089-2090.

NCD Risk Factor Collaboration. (2017). Worldwide trends in blood pressure from 1975 to 2015: a pooled analysis of 1479 population-based measurement studies with 19.1 million participants. The Lancet, 389(10064), 37-55.

Ossege, C. L., Santos, O. M. dos, Franco, A. C. D., Hoepfner, N. de O., Carvalho, D. S. B. de, Moraes, G. H., Rego, E. R. de M., Machado, A. C., \& Ronca, D. B. (2020). Atuação do profissional de saúde residente no enfrentamento da covid-19: um relato de experiência da secretaria de estado de saúde do distrito federal. Cenas Educacionais, 3, e8489.

Palmares, F. C. (2019). Fundação Palmares. www.palmares.gov.br.

Palmares, F. C. (2019). Certificação Quilombola. http://www.palmares.gov.br/?page_id=37551

Pereira, R. N., Mussi, R. F. F., \& Rocha, R. M. (2020). Acesso e utilização dos serviços de saúde por quilombolas contemporâneos baianos. Revista da ABPN, 12(31), 449-469.

Pereira, R. das N., \& Mussi, R. F. de F. (2020). Acesso e utilização dos serviços de saúde da população negra quilombola: uma análise bibliográfica. ODEERE, 5(10), 280-303.

Perini, T. A., Oliveira, G. L. de, Ornellas, J. dos S., \& Oliveira, F. P. de. (2005). Cálculo do erro técnico de medição em antropometria. Revista Brasileira de Medicina do Esporte, 11(1), 81-85.

Radovanovic, C. A. T., Santos, L. A. dos, Carvalho, M. D. de B., \& Marcon, S. S. (2014). Arterial Hypertension and other risk factors associated with cardiovascular diseases among adults. Revista Latino-Americana de Enfermagem, 22(4), 547-553.

Ribeiro, J. C. de O. A., Silva, D. O. V. da, Cardoso, E. A. M., \& Nunes, C. P. (2018). A educação do campo em tempos de privatização e golpe. Cenas Educacionais, 1(1), 111-128.

Santos, A. G., Melo, F. T., Santos, M. da S., \& Mussi, R. F. de F. (2020). Fatores associados à obesidade em adultos quilombolas baianos. RBONE - Revista Brasileira De Obesidade, Nutrição E Emagrecimento, 14(85), 230-240.

Silva, D. A. S., Petroski, E. L., \& Peres, M. A. (2012). Pré-hipertensão e hipertensão em adultos de Florianópolis: estudo de base populacional. Revista de Saúde Pública, 46(6), 988-998.

Silva, E. C., Martins, M. S. A. S., Guimarães, L. V., Segri, N. J., Lopes, M. A. L., \& Espinosa, M. M. (2016). Prevalência de hipertensão arterial sistêmica e fatores associados em homens e mulheres residentes em municípios da Amazônia Legal. Revista Brasileira de Epidemiologia, 19(1), 38-51.

Sousa, M. G. de. (2015). Tabagismo e Hipertensão arterial: como o tabaco eleva a pressão. Revista Brasileira de hipertensão, $22(3)$, 78-83.

Souza, D. do S. M. (2014). Álcool e hipertensão. Aspectos epidemiológicos, fisiopatológicos e clínicos, Revista Brasileira de Hipertensão, 21 (2), 83-86.

Stewart A, Marfell-Jones M, Olds T, Ridder H. (2011). International standards for anthropometric assesment. Lower Hutt: ISAK

Teixeira Mussi, L. M. P., Mussi, R. F. F., Araújo, A. G. (2020). Negligência/Negação à Escolarização em Quilombos da Região de Guanambi/Bahia. Veredas, $3(5), 80-103$. 
Research, Society and Development, v. 10, n. 2, e37210211055, 2021

(CC BY 4.0) | ISSN 2525-3409 | DOI: http://dx.doi.org/10.33448/rsd-v10i2.11055

US Department of Health and Human Services, National Institutes of Health. (2008). Understanding Adult Obesity. WIN Weight-control Information Network: National Institute of Diabetes and Digestive and Kidney Diseases (NIDDK) Web site. NIH Publication No. 06-3680. 2008. $<$ healthfinder.gov/FindServices/Organizations/Organization.aspx?code=H R2455>. 